\title{
Vorwort zur 11. Auflage
}

In der hiermit vorgelegten Neubearbeitung des Allgemeinen Verwaltungsrechts hat Hans-Jürgen Papier den bisher von Jürgen Salzwedel bearbeiteten 5. Abschnitt übernommen. Dieser Teil des Buches beschränkt sich nunmehr auf die Darstellung des Rechts der öffentlichen Sachen, während die Fragen der Anstaltsnutzung im 3. Abschnitt im Zusammenhang mit den verwaltungsrechtlichen Sonderverbindungen erörtert werden. Selbstverständlich ist in der Bearbeitung der einzelnen Abschnitte der Entwicklung des Gemeinschaftsrechts und seinen zunehmenden Auswirkungen auf das deutsche Verwaltungsrecht ebenso Rechnung getragen wie der Entwicklung der Gesetzgebung, der Rechtsprechung und der wissenschaftlichen Diskussion im Bereich des Allgemeinen Verwaltungsrechts und des Verwaltungsverfahrensrechts.

Jürgen Salzwedel sei auch an dieser Stelle nochmals sehr herzlich dafür gedankt, daß er über zehn Auflagen hinweg an diesem Buch mitgewirkt hat. Zugleich darf ich Herrn Assessor Christian Biermann, wissenschaftlicher Mitarbeiter am Kommunalwissenschaftlichen Institut der Westfälischen Wilhelms-Universität, sehr herzlich für die Übernahme der mit der herausgeberischen Koordination und mit der drucktechnischen Vereinheitlichung verbundenen Arbeiten danken.

In Fortsetzung und Ergänzung dieses Werkes liegt das von Eberhard SchmidtAßmann herausgegebene Lehrbuch Besonderes Verwaltungsrecht vor, das alsbald in 11. Auflage erscheinen wird.

Für Anregungen und Kritik sind Autoren und Herausgeber dankbar.

Münster, im Juli 1998

Hans-Uwe Erichsen 